ARTICLE

\title{
A top-down strategy for amorphization of hydroxyl compounds for electrocatalytic oxygen evolution
}

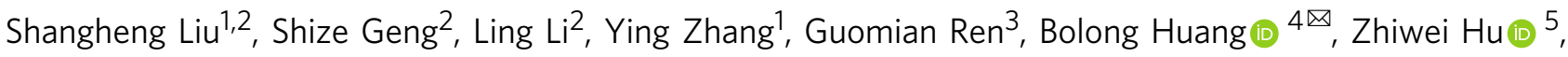

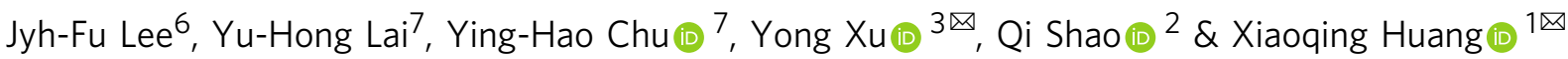

Amorphous materials have attracted increasing attention in diverse fields due to their unique properties, yet their controllable fabrications still remain great challenges. Here, we demonstrate a top-down strategy for the fabrications of amorphous oxides through the amorphization of hydroxides. The versatility of this strategy has been validated by the amorphizations of unitary, binary and ternary hydroxides. Detailed characterizations indicate that the amorphization process is realized by the variation of coordination environment during thermal treatment, where the $\mathrm{M}-\mathrm{OH}$ octahedral structure in hydroxides evolves to $\mathrm{M}-\mathrm{O}$ tetrahedral structure in amorphous oxides with the disappearance of the $\mathrm{M}-\mathrm{M}$ coordination. The optimal amorphous oxide $\left(\mathrm{FeCoSn}(\mathrm{OH})_{6}-300\right)$ exhibits superior oxygen evolution reaction (OER) activity in alkaline media, where the turnover frequency (TOF) value is 39.4 times higher than that of $\mathrm{FeCoSn}(\mathrm{OH})_{6}$. Moreover, the enhanced OER performance and the amorphization process are investigated with density functional theory (DFT) and molecule dynamics (MD) simulations. The reported top-down fabrication strategy for fabricating amorphous oxides, may further promote fundamental research into and practical applications of amorphous materials for catalysis.

\footnotetext{
${ }^{1}$ State Key Laboratory of Physical Chemistry of Solid Surfaces, College of Chemistry and Chemical Engineering, Xiamen University, 361005 Xiamen, China. ${ }^{2}$ College of Chemistry, Chemical Engineering and Materials Science, Soochow University, 215123 Jiangsu, China. ${ }^{3}$ Guangzhou Key Laboratory of LowDimensional Materials and Energy Storage Devices, Collaborative Innovation Center of Advanced Energy Materials, School of Materials and Energy, Guangdong University of Technology, 510006 Guangzhou, China. ${ }^{4}$ Department of Applied Biology and Chemical Technology, The Hong Kong Polytechnic University, Hung Hom, Kowloon, Hong Kong SAR, China. ${ }^{5}$ Max Planck Institute for Chemical Physics of Solids, Nothnitzer Strasse 40, 01187

Dresden, Germany. ${ }^{6}$ National Synchrotron Radiation Research Center, 101 Hsin-Ann Road, 30076 Hsinchu, Taiwan. ${ }^{7}$ Department of Materials Science and

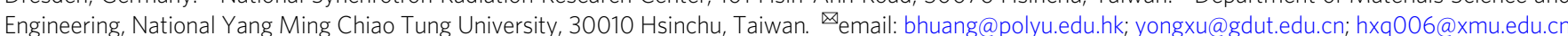


A morphous materials have attracted great attention due to their disordered atomic arrangement and unsaturated coordination environment ${ }^{1,2}$, which have been widely used in diverse fields including mechanical engineering, catalysis, and magnetic applications ${ }^{3-5}$. Compared to their crystalline analogues, amorphous materials consist of continuous random networks instead of periodic structures, and usually display some unique properties ${ }^{6-8}$. Over the past decades, substantial efforts have been devoted to the fabrications and applications of amorphous materials ${ }^{9,10}$. For example, Shao and co-workers reported that the amorphous $\mathrm{Ba}_{0.5} \mathrm{Sr}_{0.5} \mathrm{Co}_{0.8} \mathrm{Fe}_{0.2} \mathrm{O}_{3-\delta}$ nanofilms with tunable oxidation state enable up to 315 -fold enhanced massspecific activity towards oxygen evolution reaction (OER) compared to the crystalline BSCF ${ }^{11}$. Huang et al. demonstrated that the amorphization of $\mathrm{RuTe}_{2}$ resulted in the local distortion-strain effect, which could abnormally sensitize the Te-p $\pi$ coupling capability and enhance the electron transfer of Ru-sites, as a result of significant enhancement on OER performance ${ }^{12}$. Chen and coworkers demonstrated that a transformation of perovskite oxides $\left(\mathrm{ABO}_{3}\right)$ to amorphous motifs via the leaching of $\mathrm{A}$ sites or $\mathrm{B}$ sites can significantly promote the OER process ${ }^{13}$. Additionally, Zhang et al. demonstrated the lithiation-induced amorphization of layered crystalline $\mathrm{Pd}_{3} \mathrm{P}_{2} \mathrm{~S}_{8}$ can activate this otherwise electrochemically inert material into a highly efficient catalyst for hydrogen evolution reaction (HER) ${ }^{14}$. Therefore, the development of amorphous nanomaterials is of great importance in material science.

Despite these unique properties of amorphous materials, the wide-scale applications of amorphous materials still remain great challenges due to the following reasons: (1) it is still lack of facile protocols for fabricating amorphous materials; (2) High temperature and pressure may further result in recrystallization of amorphous materials, and thus the stability of amorphous materials is strongly limited by the working conditions ${ }^{15,16}$. Therefore, amorphous materials are usually synthesized and used under mild conditions (e.g., low temperature) ${ }^{17-19}$, which severely limits their practical applications. Under such circumstances, pressure-induction and high-temperature cooling strategies have been developed for the formation of amorphous materials, yet suffer from the drawbacks of complicated operation and high $\operatorname{cost}^{20,21}$. It thus highly desired to develop facile and versatile strategies for the fabrications of amorphous materials.

Inspired by the previous report that the leaching of $\mathrm{A}$ sites or $\mathrm{B}$ sites from perovskite oxides $\left(\mathrm{ABO}_{3}\right)$ can lead to the formation of amorphous motifs ${ }^{13}$, we speculate that the structural transformation may lead to the random rearrangement of atoms and thus the formation of amorphous materials. Therefore, we selected $\mathrm{CoSn}(\mathrm{OH})_{6}$, a perovskite hydroxide with poor thermal stability which can readily suffer from dehydration at high temperature 22,23 , as a model to systematically study the transformation from highly ordered structure to amorphous oxide. Experimental observations indicate that $\operatorname{CoSn}(\mathrm{OH})_{6}$ can be converted into amorphous $\mathrm{CoSnO}_{\mathrm{x}}$ oxide via a facile lowtemperature heat treatment process. Detailed characterizations reveal that the amorphization process experiences the transformation of $\mathrm{M}-\mathrm{OH}$ octahedron in $\mathrm{CoSn}(\mathrm{OH})_{6}$ into $\mathrm{M}-\mathrm{O}$ tetrahedron in amorphous oxide. Moreover, such strategy can be extended to the fabrications of other binary amorphous oxides (e.g., $\mathrm{MgSnO}_{\mathrm{x}}, \mathrm{CaSnO}_{\mathrm{x}}, \mathrm{MnSnO}_{\mathrm{x}}, \mathrm{FeSnO}_{\mathrm{x}}, \mathrm{ZnSnO}_{\mathrm{x}}$, and $\mathrm{CdSnO}_{\mathrm{x}}$ ) and ternary oxides (e.g., $\mathrm{MgCoSnO}_{\mathrm{x}}, \mathrm{CaCoSnO}_{\mathrm{x}}, \mathrm{MnCoSnO}_{\mathrm{x}}$, $\mathrm{FeCoSnO}_{\mathrm{x}}, \mathrm{NiCoSnO}_{\mathrm{x}}, \mathrm{CuCoSnO}_{\mathrm{x}}, \mathrm{CdCoSnO}_{\mathrm{x}}$, and $\mathrm{ZnCoSnO}_{\mathrm{x}}$ ), being a versatile strategy for the formation of amorphous materials. The optimal amorphous oxide (e.g., $\mathrm{FeCoSn}(\mathrm{OH})_{6}-300$ ) exhibits promising oxygen evolution reaction (OER) performance in terms of high activity and stability in alkaline media. This work provides a versatile top-down strategy for fabricating amorphous oxides, which may further promote the fundamental researches and practical applications of amorphous materials for catalysis.

\section{Results}

Synthesis and characterization. $\operatorname{CoSn}(\mathrm{OH})_{6}$ was synthesized via a precipitation method at room temperature (see details in experimental section). High-angle annular dark-field scanning transmission electron microscopy (HAADF-STEM) image shows that the obtained $\operatorname{CoSn}(\mathrm{OH})_{6}$ perovskite hydroxide has a morphology of cube with a mean size is $\sim 50 \mathrm{~nm}$ (Fig. 1a). The energydisperse X-ray spectroscopy (EDS) profile implies that the Co:Sn is close to 1 (Supplementary Fig. 1). After thermal treatment at $300{ }^{\circ} \mathrm{C}$ (named as $\mathrm{CoSn}(\mathrm{OH})_{6}-300$ ), no obvious variations in the morphology and size are observed (Fig. 1b). The characteristic peaks in the X-ray diffraction (XRD) pattern are ascribed to $\mathrm{CoSn}(\mathrm{OH})_{6}$ perovskite hydroxide (PDF: 13-0356) (Fig. 1c). By sharp contrast, the absence of peaks in the XRD pattern suggests that $\operatorname{CoSn}(\mathrm{OH})_{6}-300$ is amorphous (Fig. 1d). High-resolution TEM (HRTEM) image further confirms the transformation from highly crystalline to amorphous structure (Fig. 1e, f), as evidenced by the evolutions of diffraction patterns (inset of Fig. 1e, f). The as-prepared $\mathrm{CoSn}(\mathrm{OH})_{6}$ with cubic structure (space group $\mathrm{Pn}_{3} \mathrm{~m} /$ 224) is confirmed by the selected area electron diffraction (SAED) pattern along the [011] zone axis (inset of Fig. 1e), and the diffraction dots of the (200), (220) and (020) planes are observed. By contrast, the corresponding diffraction halo in SAED pattern confirms the amorphous properties of the materials. Moreover, infrared (IR) and Raman spectroscopy were employed to reveal the amorphization process of $\operatorname{CoSn}(\mathrm{OH})_{6}$. As shown in Fig. 1g, the characteristic peak of $\mathrm{M}-\mathrm{OH}$ bond in $\operatorname{CoSn}(\mathrm{OH})_{6}$ at $1200 \mathrm{~cm}^{-1}$ disappears after thermal treatment, which might be attributed to the conversion of $\mathrm{M}-\mathrm{OH}$ bond to $\mathrm{M}-\mathrm{O}$ bond ${ }^{24}$. Similar phenomena are observed in the Raman spectra of the $\mathrm{CoSn}(\mathrm{OH})_{6}$ and $\mathrm{CoSn}(\mathrm{OH})_{6}-300$. The four bands at 304-433 and $602 \mathrm{~cm}^{-1}$ in the Raman spectrum of $\mathrm{CoSn}(\mathrm{OH})_{6}$ are attributed to the breathing vibration of the long $\mathrm{M}-\mathrm{OH}$ bonds and the bending mode of $\mathrm{M}-\mathrm{OH}-\mathrm{M}$ (bridging $\mathrm{OH}$ group), respectively ${ }^{25}$. After amorphization, a broad band appears at $500-750 \mathrm{~cm}^{-1}$ in the Raman spectrum of $\operatorname{CoSn}(\mathrm{OH})_{6}-300$, which may be attributed to the distorted structure of $\operatorname{CoSn}(\mathrm{OH})_{6}-300$ after the transformation from $\mathrm{M}-\mathrm{O}$ octahedron to $\mathrm{M}-\mathrm{O}$ tetrahedron ${ }^{26}$. In addition, X-ray photoelectron spectroscopy (XPS) measurement was conducted to investigate the surface properties of $\mathrm{CoSn}(\mathrm{OH})_{6}$ and $\mathrm{CoSn}(\mathrm{OH})_{6}$ 300. No obvious changes are observed in the Co $2 p$ and Sn $3 d$ XPS spectra $^{27,28}$. The peaks at 781.1 and $785.6 \mathrm{eV}$ in the of Co $2 p$ XPS spectra are ascribed to $\mathrm{Co}^{2+}$ and the satellite, respectively. The similar features in the XPS spectra of $\mathrm{CoSn}(\mathrm{OH})_{6}$ and $\mathrm{CoS}$ $\mathrm{n}(\mathrm{OH})_{6}-300$ imply the valence states of Co are similar before and after amorphization (i.e., $\left.\mathrm{Co}^{2+}\right)^{29}$. Compared to $\mathrm{CoSn}(\mathrm{OH})_{6}$, the peak position in the $\mathrm{O} 1 s$ XPS spectrum of $\mathrm{CoSn}(\mathrm{OH})_{6}-300$ negatively shifts by $1.2 \mathrm{eV}$, which is attributed to the formation of $\mathrm{M}-\mathrm{O}$ bond in $\mathrm{CoSn}(\mathrm{OH})_{6}-300$ (Fig. 1k) ${ }^{30}$.

Mechanism studies for amorphization. To understand the process and mechanism of amorphization, thermogravimetric (TG) measurement was performed for $\operatorname{CoSn}(\mathrm{OH})_{6}$ in $\operatorname{argon}(\mathrm{Ar})$ to study the weight loss during thermal treatment. It is found that $\mathrm{CoSn}(\mathrm{OH})_{6}$ experiences a gradual weight loss at $30-240{ }^{\circ} \mathrm{C}$ (Supplementary Fig. 2a) ${ }^{31}$. Two intense peaks appear at $\sim 180$ and $\sim 240^{\circ} \mathrm{C}$ in the derivative thermogravimetric (DTG) curve (Supplementary Fig. 2b), which can be attributed to the dehydration of $\mathrm{CoSn}(\mathrm{OH})_{6}$. Moreover, we measured the weight losses by heating $\mathrm{CoSn}(\mathrm{OH})_{6}$ to 150 and $250^{\circ} \mathrm{C}$ for $1 \mathrm{~h}$ with a rate of $10^{\circ} \mathrm{C} / \mathrm{min}$ (Supplementary Fig. 3). It is found that $\mathrm{CoSn}(\mathrm{OH})_{6}$ experiences a gradual weight loss when treated at $150{ }^{\circ} \mathrm{C}$. For $\operatorname{CoSn}(\mathrm{OH})_{6}$ 

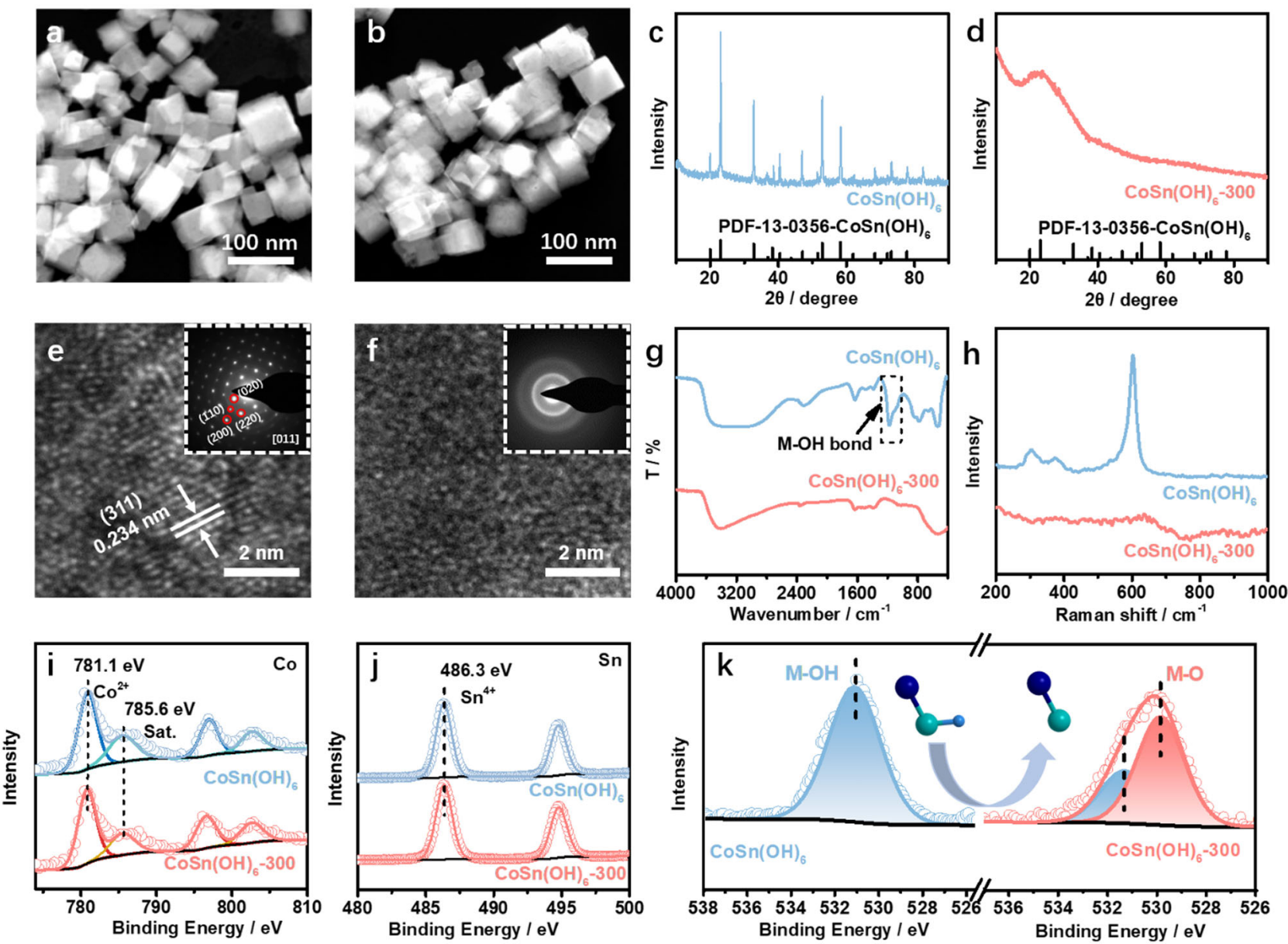

Fig. 1 Structural analysis of $\operatorname{CoSn}(\mathbf{O H})_{6}$ before and after amorphization. HAADF-STEM images of $\mathbf{a} \operatorname{CoSn}(\mathrm{OH})_{6}$ and $\mathbf{b} \operatorname{CoSn}(\mathrm{OH})_{6}-300$. XRD patterns of c $\mathrm{CoSn}(\mathrm{OH})_{6}$, and $\mathbf{d} \mathrm{CoSn}(\mathrm{OH})_{6}-300$. HRTEM images of $\mathbf{e} \mathrm{CoSn}(\mathrm{OH})_{6}$ and $\mathbf{f} \mathrm{CoSn}(\mathrm{OH})_{6}-300$. Inset of $\mathbf{e}$ and $\mathbf{f}$ are the corresponding SAED patterns. $\mathbf{g}$ FTIR and $\mathbf{h}$ Raman spectra of $\mathrm{CoSn}(\mathrm{OH})_{6}$ and $\mathrm{CoSn}(\mathrm{OH})_{6}-300$. $\mathbf{i} \mathrm{Co} 2 p, \mathbf{j}$ Sn $3 d$, and $\mathbf{k} \mathrm{O} 1 \mathrm{~s}$ XPS spectra of $\mathrm{CoSn}(\mathrm{OH})_{6}$ and $\mathrm{CoSn}(\mathrm{OH})_{6}-300$.

treated at $250^{\circ} \mathrm{C}$, it suffers from a rapid weight loss during the heating process, while no obvious weight losses were observed in the next $1 \mathrm{~h}$ at the target temperatures, being consistent with above TG analysis. Furthermore, XRD patterns of thermally treated $\mathrm{CoSn}(\mathrm{OH})_{6}$ at different temperatures were collected. As shown in Fig. 2a, the characteristic peaks of $\mathrm{CoSn}(\mathrm{OH})_{6}$ were reserved in the XRD pattern when $\mathrm{CoSn}(\mathrm{OH})_{6}$ were treated at 100 and $150^{\circ} \mathrm{C}$ (named as $\operatorname{CoSn}(\mathrm{OH})_{6}-100$ and $\operatorname{CoSn}(\mathrm{OH})_{6}-150$, respectively). When the temperature was increased to $175^{\circ} \mathrm{C}$ $\left(\mathrm{CoSn}(\mathrm{OH})_{6}-175\right)$, the peaks in the XRD patterns are strongly weakened, which completely disappear when the temperature for thermal treatment is over $200^{\circ} \mathrm{C}$, further confirming the amorphization of $\mathrm{CoSn}(\mathrm{OH})_{6}$. X-ray absorption near-edge structure spectroscopy (XANES) spectra at the Co- $K$ edge were collected to investigate the structures. As shown in Fig. $2 \mathrm{~b}$, the Co- $K$-edge XANES spectra of the treated $\operatorname{CoSn}(\mathrm{OH})_{6}$ at different temperatures display similar features to that of $\mathrm{CoO}$ reference ${ }^{32-35}$, indicating that $\mathrm{Co}$ in treated $\mathrm{CoSn}(\mathrm{OH})_{6}$ present as $\mathrm{Co}^{2+}$. Beisides, the electronic structures were studied by soft X-ray absorption spectroscopy (XAS) at the Co- $L_{2,3}$ edges, which are highly sensitive to the valence state, spin state environment and local environment $t^{36-40}$. Figure $2 c$ shows that the energy position and mutliple spectral features of the Co-L-edge XAS spectra of $\mathrm{CoSn}(\mathrm{OH})_{6}$. No obvious changes of the valence states of $\mathrm{Co}$ (i.e., $\mathrm{Co}^{2+}$ ) are observed during the thermal treatment at different temperatures, whereas the local symmetry changes with the increased temperature for thermal treatment, as revealed by the multiple spectral features (Fig. 2c). The sharp peak at $777.8 \mathrm{eV}$ in the $\mathrm{Co}-L_{3}$ edge of $\mathrm{CoO}$ (labelled in Fig. 2c) can be ascribed to $\mathrm{Co}^{2+}$ with octahedral coordination. The peak intensity decreases continuously with the increased temperature, suggesting the transformation of $\mathrm{Co}^{2+}$ octahedral coordination to tetrahedral coordination ${ }^{41,42}$. In the extended X-ray absorption fine structure (EXAFS) spectra, the peaks at 1.51 and $2.94 \AA$ for $\mathrm{CoSn}(\mathrm{OH})_{6}$ are ascribed to $\mathrm{Co}-\mathrm{OH}$ and $\mathrm{Co}-\mathrm{Sn}$ coordination, respectively (Fig. 2d $)^{43}$. As increasing the temperature for thermal treatment (over $200^{\circ} \mathrm{C}$ ), the gradual weakening of $\mathrm{Co}-\mathrm{OH}$ coordination and the presence of $\mathrm{Co}-\mathrm{O}$ coordination in the EXAFS spectra of $\operatorname{CoSn}(\mathrm{OH})_{6}-200$ and $\mathrm{CoSn}(\mathrm{OH})_{6}-300$ suggest the structural evolution. Moreover, the absence of Co-Sn coordination at $2.94 \AA$ suggests the formation of disordered structure ${ }^{44}$. The average coordination number $(\mathrm{CN})$ value derived from the least-squares curve fitting of 1st $\mathrm{Co}-\mathrm{OH}$ shell was used to reveal the structure of treated $\mathrm{CoSn}(\mathrm{OH})_{6}$ (Supplementary Fig. 4) ${ }^{45,46}$. The structural evolutions during the thermal treatment are further validated by the variations of $\mathrm{CN}$ (Fig. 2e and Supplementary Table 1). In particular, the slight decrease of $\mathrm{CN}$ of $\mathrm{Co}-\mathrm{OH}$ in the treated samples at $150-175^{\circ} \mathrm{C}$ is attributed to the dehydration of CoS$n(\mathrm{OH})_{6}$. After the transformation from $\mathrm{Co}-\mathrm{OH}$ octahedron to $\mathrm{Co}-\mathrm{O}$ tetrahedron, the $\mathrm{CN}$ of $\mathrm{Co}-\mathrm{O}$ deceases to 4.2 for CoS$\mathrm{n}(\mathrm{OH})_{6}-300$ (Supplementary Table 1 ). Sepecifically, The $\mathrm{CN}$ of $\mathrm{CoSn}(\mathrm{OH})_{6}-300$ is similar to that $\mathrm{CoO}$ (cubic, F-43m [216]). In addition, wavelet transform (WT) contour plots of $\mathrm{CoSn}(\mathrm{OH})_{6}$ and $\operatorname{CoSn}(\mathrm{OH})_{6}-300$ have further validated the structural 

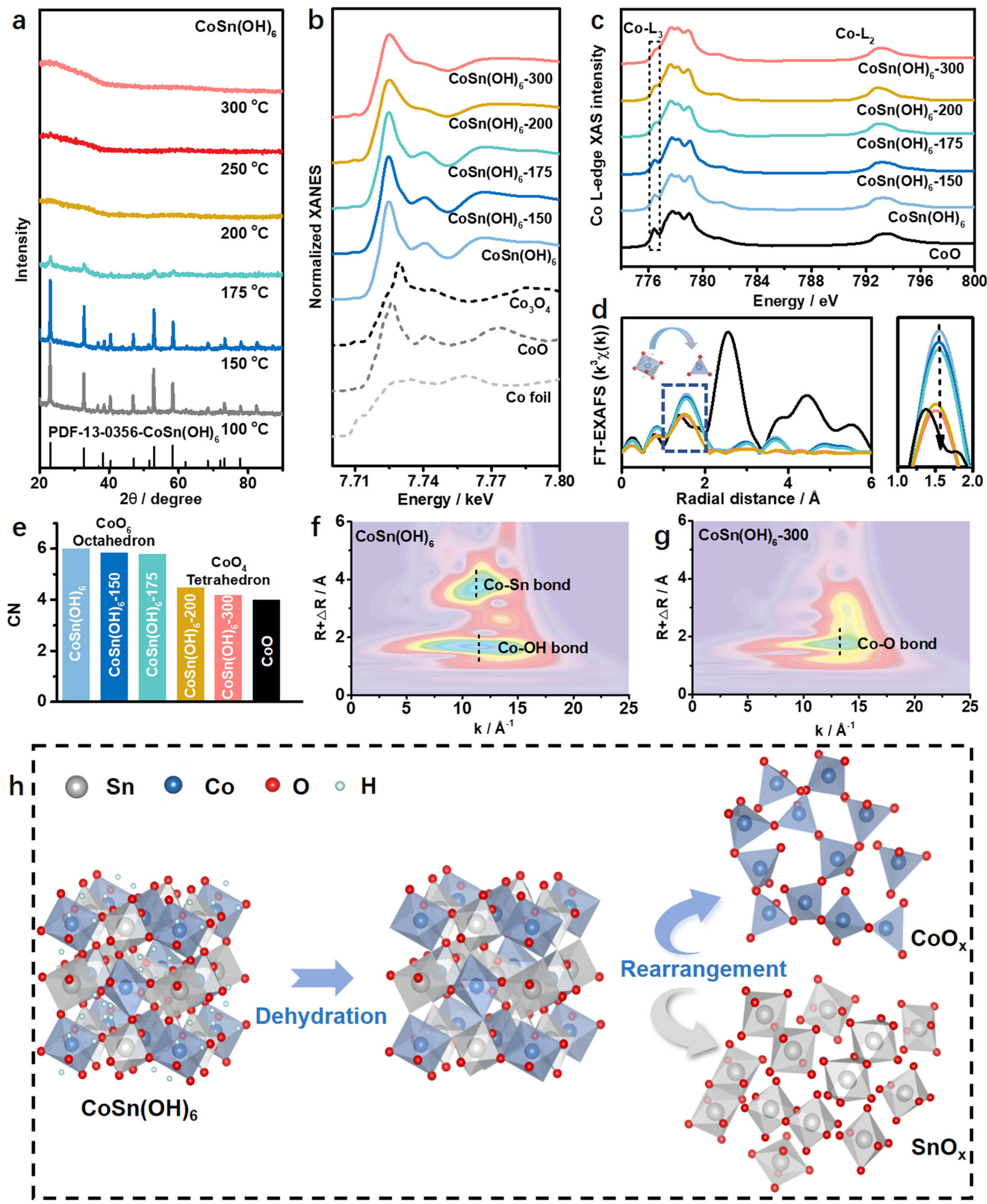

Fig. 2 Characterizations for the amorphization. a XRD patterns collected after treating $\operatorname{CoSn}(\mathrm{OH})_{6}$ at different temperatures. b Normalized XANES spectra of various samples at Co- $K$ edge. c Co-L-edge XAS spectra of pristine $\mathrm{CoSn}(\mathrm{OH})_{6}$ and $\mathrm{CoSn}(\mathrm{OH})_{6}$ treated at different temperatures. $\mathbf{d}$ Fourier transforms of Co-K-edge EXAFS spectra (left) and enlarged spectra at 1-2 $\AA$ (right) of different samples. The colours in $\mathbf{d}$ are same with those in $\mathbf{b}$. e The average coordination numbers (CNs) of different samples obtained from the fitting of EXAFS spectra. Wavelet transform of Co-K-edge EXAFS data of $\mathbf{f}$ $\mathrm{CoSn}(\mathrm{OH})_{6}$ and $\mathbf{g} \mathrm{CoSn}(\mathrm{OH})_{6}-300$. $\mathbf{h}$ Schematic illustration for the structural evolution of $\mathrm{CoSn}(\mathrm{OH})_{6}$ during amorphization. 
transformation from $\mathrm{M}-\mathrm{OH}$ to $\mathrm{M}-\mathrm{O}$ coordination (Fig. 2f, g). Compared to the WT contour plot of $\mathrm{CoSn}(\mathrm{OH})_{6}$ with two features of $\mathrm{Co}-\mathrm{OH}$ to $\mathrm{Co}-\mathrm{Sn}$ coordination, only one band corresponding to $\mathrm{Co}-\mathrm{O}$ appears in the WT contour plot of $\mathrm{CoSn}(\mathrm{OH})_{6}-300$, indicating the transformation from $\mathrm{Co}-\mathrm{OH}$ to $\mathrm{Co}-\mathrm{O}$ coordination in $\mathrm{CoSn}(\mathrm{OH})_{6}-300$. Moreover, the absence of $\mathrm{Co}-\mathrm{Sn}$ coordination further implies the disordered structure of $\mathrm{CoSn}(\mathrm{OH})_{6}-300$. Based on the above analysis, we conclude the structural transformation process for $\mathrm{CoSn}(\mathrm{OH})_{6}$. Specifically, $\mathrm{Co}-\mathrm{OH}$ octahedron evolves to $\mathrm{Co}-\mathrm{O}$ tetrahedron during the decomposition of $\mathrm{CoSn}(\mathrm{OH})_{6}$ at high temperature, and the 2nd $\mathrm{Co}-\mathrm{Sn}$ coordination disappears once $\mathrm{Co}-\mathrm{O}$ coordination is generated, leading to the formation of disordered structure (Fig. $2 \mathrm{~h}$ ).

To further study the amorphization of $\operatorname{CoSn}(\mathrm{OH})_{6}$, the physicochemical properties of $\mathrm{CoSn}(\mathrm{OH})_{6}-300$ were investigated. Compared to the pristine $\mathrm{CoSn}(\mathrm{OH})_{6}, \mathrm{CoSn}(\mathrm{OH})_{6}-300$ displays a much higher surface area of $158 \mathrm{~m}^{2} \mathrm{~g}^{-1}$ (Supplementary Fig. 5), indicating the formation of porous structure after amorphization ${ }^{47}$. It is noted that the amorphous structure is maintained even at a treatment temperature up to $500^{\circ} \mathrm{C}$ (Supplementary Fig. 6), suggesting the enhanced thermal stability of the amorphous oxides. Moreover, the morphology, size, and structure of the obtained amorphous structures are largely maintained after scaling up to gram level, showing the great potential of this top-down strategy for the formation of amorphous structures (Supplementary Fig. 7). Furthermore, unitary hydroxide, binary, and ternary perovskite hydroxides were synthesized to demonstrate the versatility of this top-down strategy for the fabrications of amorphous structures. As depicted in Supplementary Figs. 8 and 9, it is found that two-dimensional $\mathrm{Co}(\mathrm{OH})_{2}$ and $\mathrm{Cd}(\mathrm{OH})_{2}$ nanosheets can be converted into amorphous oxides after thermal treatments in $\mathrm{Ar}$ at $400^{\circ} \mathrm{C}$ for $1 \mathrm{~h}$. Moreover, various binary perovskite hydroxides including $\mathrm{ZnSn}(\mathrm{OH})_{6}, \mathrm{CdSn}(\mathrm{OH})_{6}, \mathrm{FeSn}(\mathrm{OH})_{6}, \mathrm{MnSn}(\mathrm{OH})_{6}, \mathrm{CaSn}(\mathrm{OH})_{6}$, and $\mathrm{MgSn}(\mathrm{OH})_{6}$ were synthesized (Supplementary Fig. 10). With similar manner, these binary perovskite hydroxides experience amorphization to form corresponding binary amorphous oxides (Fig. 3a, b). Besides, the generality of this top-down strategy has been validated by the amorphization of $\mathrm{CdIr}(\mathrm{OH})_{6}$, a Sn-free binary hydroxide (Supplementary Fig. 11). Additionally, ternary perovskite hydroxides, such as $\mathrm{ZnCoSn}(\mathrm{OH})_{6}, \mathrm{CdCoSn}(\mathrm{OH})_{6}$, $\mathrm{CuCoSn}(\mathrm{OH})_{6}, \mathrm{NiCoSn}(\mathrm{OH})_{6}, \quad \mathrm{FeCoSn}(\mathrm{OH})_{6}, \mathrm{MnCoSn}(\mathrm{OH})_{6}$, $\mathrm{CaCoSn}(\mathrm{OH})_{6}$, and $\mathrm{MgCoSn}(\mathrm{OH})_{6}$ (Supplementary Fig. 12), can also be transformed into amorphous oxides through this topdown strategy (Fig. 3c-e). We take $\mathrm{FeCoSn}(\mathrm{OH})_{6}$ as an example, SEM and TEM images indicate that ternary $\mathrm{FeCoSn}(\mathrm{OH})_{6}$ perovskite hydroxide has a spherical shape with a mean size of $100 \mathrm{~nm}$ (Supplementary Fig. 13a, b). Elemental mapping image and line scan profile suggest that all the elements are evenly distributed in the nanospheres (Supplementary Fig. 13c, d). The surface area of ternary $\mathrm{FeCoSn}(\mathrm{OH})_{6}$ perovskite hydroxide is $62.0 \mathrm{~m}^{2} \mathrm{~g}^{-1}$ (Supplementary Fig. 13e). After thermal treatment, no obvious changes of the morphology, size, and elemental distributions are observed for the amorphous oxide, and the disappearance of lattice fringes in HRTEM image confirms the amorphization of $\mathrm{FeCoSn}(\mathrm{OH})_{6}$ (Supplementary Fig. 14a-d). Correspondingly, the surface area significantly increases to $163.6 \mathrm{~m}^{2} \mathrm{~g}^{-1}$ after amorphization (Supplementary Fig. 14e). The $\mathrm{Sn} 3 d$ and Co $2 p$ XPS spectra of the amorphous $\mathrm{FeCoSn}(\mathrm{OH})_{6}-300$ display similar features to the pristine $\mathrm{FeCoSn}(\mathrm{OH})_{6}$, whereas the intensity of $\mathrm{M}-\mathrm{O}$ in the XPS spectrum of $\mathrm{FeCoSn}(\mathrm{OH})_{6}-300$ is much higher than that of $\mathrm{FeCoSn}(\mathrm{OH})_{6}$, which is ascribed to $\mathrm{FeCoSn}(\mathrm{OH})_{6}$ dehydration during thermal treatment (Supplementary Fig. 15). XANES spectra of $\mathrm{FeCoSn}(\mathrm{OH})_{6}$ show that the valence states of $\mathrm{Fe}$ and Co are close to $\mathrm{Fe}^{3+}$ and $\mathrm{Co}^{2+}$, respectively (blue spectra in
Fig. 3f, g). Under the treatment in $\mathrm{H}_{2} / \mathrm{Ar}\left(5\right.$ vol.\%) at $300^{\circ} \mathrm{C}$ for $1 \mathrm{~h}$, the chemical valences of $\mathrm{Fe}^{3+}$ and $\mathrm{Co}^{2+}$ are largely maintained, as revealed by the similar features in Fe $K$-edge and Co-K-edge XANES spectra (red spectra in Fig. 3f, g). Besides, the variations of local electronic properties after amorphization have been further validated by the Fourier-transformed EXAFS spectra. As shown in Fig. $3 \mathrm{~h}, \mathrm{i}$, the presence of $\mathrm{Fe}-\mathrm{O}(\mathrm{Co}-\mathrm{O})$ coordination and the absence of $\mathrm{Fe}-\mathrm{OH}(\mathrm{Co}-\mathrm{OH})$ and 2nd Fe-M (Co-M) coordination in the EXAFS spectra of $\mathrm{FeCoSn}(\mathrm{OH})_{6}-300$ confirm the amorphization of $\mathrm{FeCoSn}(\mathrm{OH})_{6}$ after the treatment. Detailed analysis indicates that the $\mathrm{CN}$ of $\mathrm{Fe}-\mathrm{OH}$ and $\mathrm{Co}-\mathrm{OH}$ in $\mathrm{FeCoSn}(\mathrm{OH})_{6}$ are 5.0 and 5.6, respectively. After the thermal treatment, the $\mathrm{CNs}$ of $\mathrm{Fe}-\mathrm{O}$ and $\mathrm{Co}-\mathrm{O}$ in $\mathrm{FeCoSn}(\mathrm{OH})_{6}-300$ decrease to 3.9 and 4.4, respectively, suggesting that the octahedral $\mathrm{Fe}-\mathrm{OH}$ and $\mathrm{Co}-\mathrm{OH}$ coordination are transformed into tetrahedral $\mathrm{Fe}-\mathrm{O}$ and $\mathrm{Co}-\mathrm{O}$ coordination (Supplementary Figs. 16, 17 and Supplementary Table 2). The absence of M-M $(\mathrm{M}=\mathrm{Fe}, \mathrm{Co}$, and $\mathrm{Sn})$ coordination in the EXAFS spectrum of FeCoSn $(\mathrm{OH})_{6}-300$ further confirms the disordered structure of $\mathrm{FeCoSn}(\mathrm{OH})_{6}-300$. In addition, the versatility of such top-down strategy has been validated by fabricating $\mathrm{FeCoSn}(\mathrm{OH})_{6}$ with different compositions. As shown in Supplementary Fig. 18, as increasing the $\mathrm{Fe} / \mathrm{Co}$ ratio in $\mathrm{FeCoSn}(\mathrm{OH})_{6}$, all the products display similar XRD patterns, indicating that Fe can occupy the position of Co to form a polynary perovskite hydroxide. Note that the positive peak shifts is attributed to the lattice contraction due to the increase of $\mathrm{Fe} / \mathrm{Co}$ ratio.

Catalytic performance of amorphous oxides for OER. To study the catalytic performance of the obtained amorphous oxides, oxygen evolution reaction (OER) was performed in $\mathrm{O}_{2}$-saturated $1.0 \mathrm{M} \mathrm{KOH}$ solution. It is found that the amorphous FeCoSn $(\mathrm{OH})_{6}-300$ exhibits superior OER performance to other amorphous oxides. Specifically, the current density reaches $178 \mathrm{~mA} \mathrm{~cm}^{-2}$ at $1.6 \mathrm{~V}$ (vs. RHE) for $\mathrm{FeCoSn}(\mathrm{OH})_{6}-300$, which is significantly higher than those of other amorphous oxides (Fig. 4a and Supplementary Fig. 19). On the other hand, the OER activity of $\mathrm{FeCoSn}(\mathrm{OH})_{6}-300$ is 39.4 times higher than that of the $\mathrm{FeCoSn}(\mathrm{OH})_{6}$, indicating that the amorphization can significantly improve the OER performance (Fig. 4a). To further demonstrate the significance of amorphization on OER, $\mathrm{FeCoSn}(\mathrm{OH})_{6}$ was further converted into crystalline $\mathrm{Fe}-\mathrm{CoO} / \mathrm{SnO}_{2}$ and $\mathrm{Fe}-\mathrm{CoSn}$ alloy by annealing at $700^{\circ} \mathrm{C}$ in Air and $\mathrm{H}_{2} / \mathrm{Ar}$, respectively (Supplementary Figs. 20 and 21). As shown in Fig. 4b, the overpotentials for $\mathrm{FeCoSn}(\mathrm{OH})_{6}$, Fe-CoSn alloy and $\mathrm{Fe}-\mathrm{CoO} /$ $\mathrm{SnO}_{2}$ at $10 \mathrm{~mA} \mathrm{~cm}^{-2}$ are 342,291 , and $299 \mathrm{mV}$, respectively, which are much higher than that of $\mathrm{FeCoSn}(\mathrm{OH})_{6}-300(266 \mathrm{mV})$ under the same conditions. Moreover, the Tafel slope of

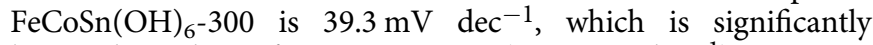
lower than that of $\mathrm{Fe}-\mathrm{CoO} / \mathrm{SnO}_{2}\left(52.4 \mathrm{mV} \mathrm{dec}{ }^{-1}\right), \mathrm{Fe}-\mathrm{CoSn}$ $\left(42.3 \mathrm{mV} \mathrm{dec}^{-1}\right)$, and $\mathrm{FeCoSn}(\mathrm{OH})_{6}\left(53.5 \mathrm{mV} \mathrm{dec}^{-1}\right)$ (Fig. 4c). Results from electrochemical active surface area (ECSA) measurement indicate that the amorphous $\mathrm{FeCoSn}(\mathrm{OH})_{6}-300$ exhibits superior OER activity to $\mathrm{FeCoSn}(\mathrm{OH})_{6}$, Fe-CoSn, Fe-CoO/SnO ${ }_{2}$, and $\mathrm{FeCoSn}(\mathrm{OH})_{6}$ (Supplementary Figs. 22 and 23) ${ }^{48}$. Nyquist plots imply that $\mathrm{FeCoSn}(\mathrm{OH})_{6}-300$ has a smaller interface chargetransfer resistance than those of other references (Supplementary Fig. 24) ${ }^{49}$. Besides, we calculated the turnover frequency (TOF) values based on the surface atoms. As shown in Fig. 4d, the TOF value of $\mathrm{FeCoSn}(\mathrm{OH})_{6}-300$ at $300 \mathrm{mV}$ is $0.622 \mathrm{~s}^{-1}$, which is $\sim 9.7$, $\sim 2.6$, and $\sim 2.6$ times higher than that of $\mathrm{FeCoSn}(\mathrm{OH})_{6}$, Fe-CoSn alloy, and $\mathrm{Fe}-\mathrm{CoO} / \mathrm{SnO}_{2}$, respectively. Additionally, cyclic voltammograms (CV) was used to evaluate the stability of FeCoS$n(\mathrm{OH})_{6}-300$ for OER, where $\mathrm{FeCoSn}(\mathrm{OH})_{6}-300$ exhibits promising stability without decrease of OER activity after 

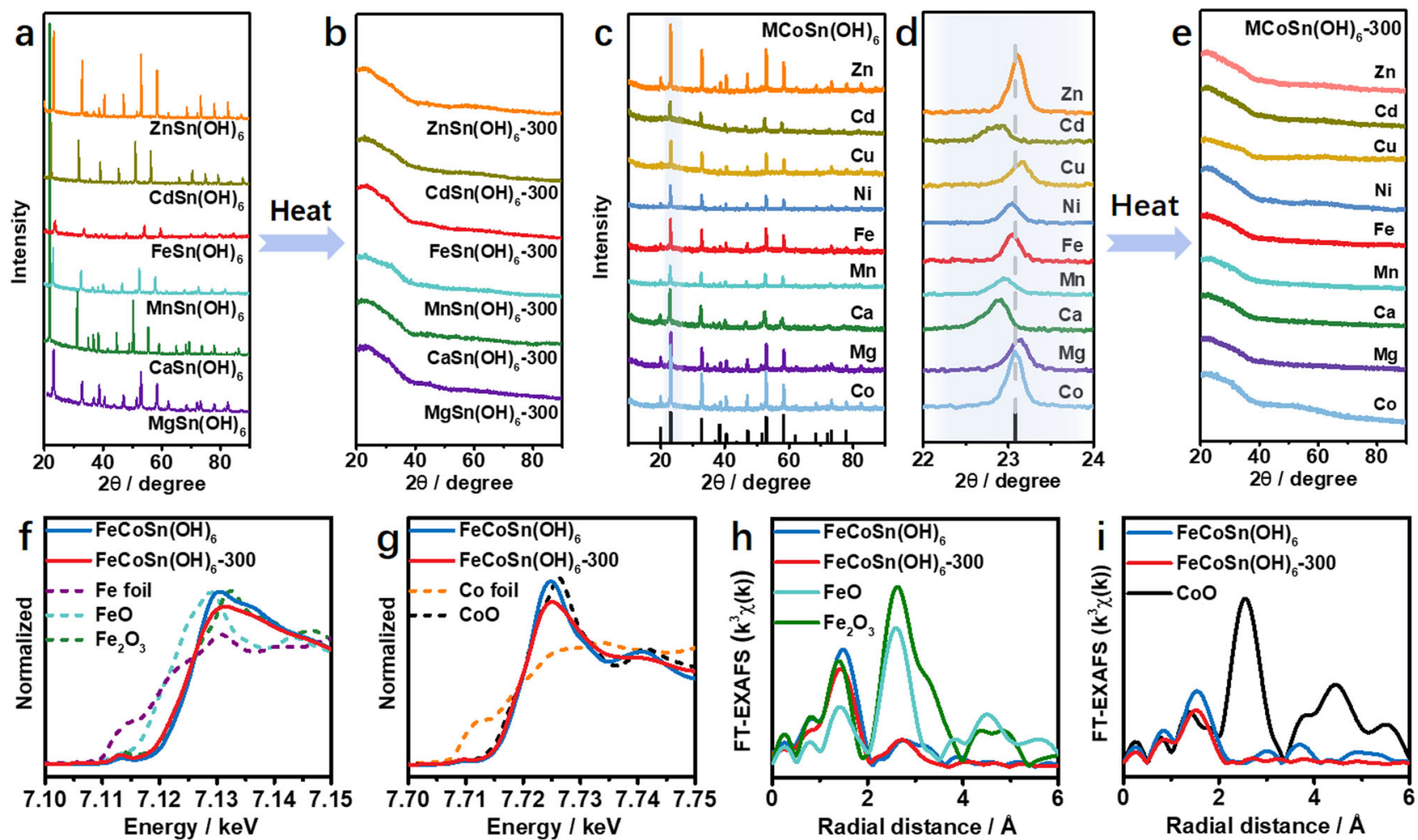

Fig. 3 Structural analysis of binary $\mathbf{M S n}(\mathrm{OH})_{6}$ and ternary $\mathbf{M C o S n}(\mathbf{O H})_{6}$ before and after amorphization. XRD patterns of a $M \mathrm{MSn}(\mathrm{OH})_{6}, \mathbf{b} \mathrm{MSn}(\mathrm{OH})_{6}$ 300 , c, d MCoSn $(\mathrm{OH})_{6}$, and e $\mathrm{MCoSn}(\mathrm{OH})_{6}$-300. f Normalized Fe $\mathrm{K}$-edge XANES spectra of FeCoSn $(\mathrm{OH})_{6}, \mathrm{FeCoSn}(\mathrm{OH})_{6}-300, \mathrm{Fe}$ foil, $\mathrm{FeO}$, and Fe $2 \mathrm{O}_{3}$. g Normalized Co-K-edge XANES spectra of $\mathrm{FeCoSn}(\mathrm{OH})_{6}, \mathrm{FeCoSn}(\mathrm{OH})_{6}-300$, Co foil, and $\mathrm{CoO}$. h Fourier transforms of EXAFS spectra of FeCoSn $(\mathrm{OH})_{6}$, $\mathrm{FeCoSn}(\mathrm{OH})_{6}-300, \mathrm{FeO}$, and $\mathrm{Fe}_{2} \mathrm{O}_{3}$ at $\mathrm{Fe}-\mathrm{K}$ edge. i Fourier transforms of EXAFS spectra of $\mathrm{FeCoSn}(\mathrm{OH})_{6}, \mathrm{FeCoSn}(\mathrm{OH})_{6}-300$, and $\mathrm{CoO}$ at $\mathrm{Co}-\mathrm{K}$-edge.
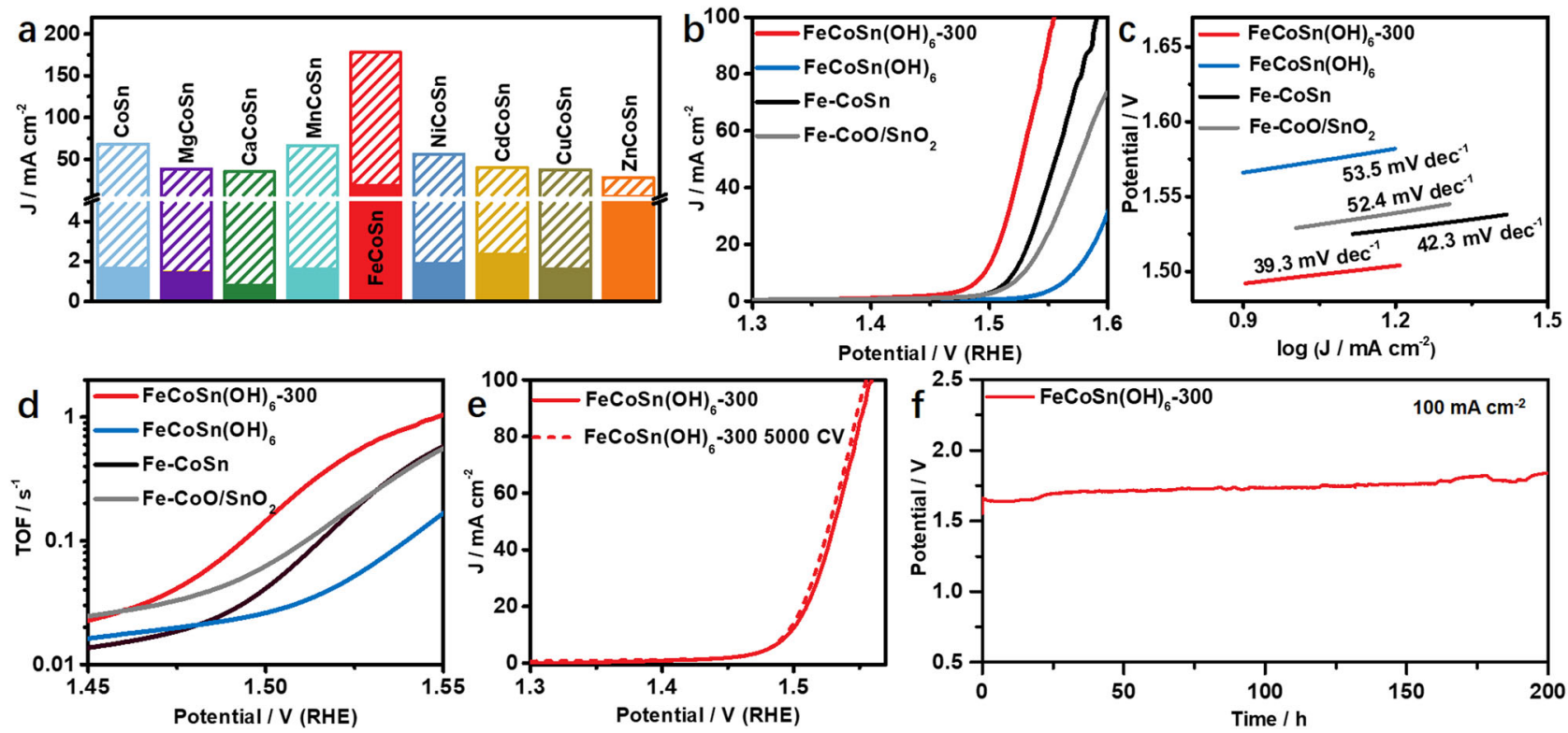

Fig. 4 Catalytic performance of OER. a The current densities of $\mathrm{MCOSn}(\mathrm{OH})_{6}$ and $\mathrm{MCOSn}(\mathrm{OH})_{6}-300$ in $\mathrm{O}_{2}$-saturated $1.0 \mathrm{M} \mathrm{KOH}$ solution. b OER polarization curves of $\mathrm{FeCoSn}(\mathrm{OH})_{6}-300, \mathrm{FeCoSn}(\mathrm{OH})_{6}, \mathrm{Fe}-\mathrm{CoSn}$, and $\mathrm{Fe}-\mathrm{Co} / \mathrm{SnO}_{2}$. c Tafel slopes of different catalysts. d TOF values collected at different potentials for $\mathrm{FeCoSn}(\mathrm{OH})_{6}-300$, $\mathrm{FeCoSn}(\mathrm{OH})_{6}, \mathrm{Fe}-\mathrm{CoSn}$, and $\mathrm{Fe}-\mathrm{Co} / \mathrm{SnO}_{2}$. e OER polarization curves of $\mathrm{FeCoSn}(\mathrm{OH})_{6}-300$ after $5000 \mathrm{cycles}$. f Continuous OER chronopotentiometry test for $\mathrm{FeCoSn}(\mathrm{OH})_{6}-300$ at $100 \mathrm{~mA} \mathrm{~cm}^{-2}$.

5000 cycles (Fig. 4e). No obvious increase of OER potential during chronopotentiometric (CP) experiment at $100 \mathrm{~mA} \mathrm{~cm}^{-2}$ for $200 \mathrm{~h}$ indicates the excellent stability of $\mathrm{FeCoSn}(\mathrm{OH})_{6}-300$ (Fig. 4f). The morphology and amorphous structure of the spent $\mathrm{FeCoSn}(\mathrm{OH})_{6}-300$ catalyst are largely maintained after OER test
(Supplementary Fig. 25a-c). Note that the dissolution of Sn can lead to the formation of defects and enhance the catalytic performance (Supplementary Fig. 25d) ${ }^{50}$. It is found that Co on the surface of amorphous $\mathrm{FeCoSn}(\mathrm{OH})_{6}-300$ is slightly oxidized into $\mathrm{CoOOH}$ after activation and stability test (Supplementary 

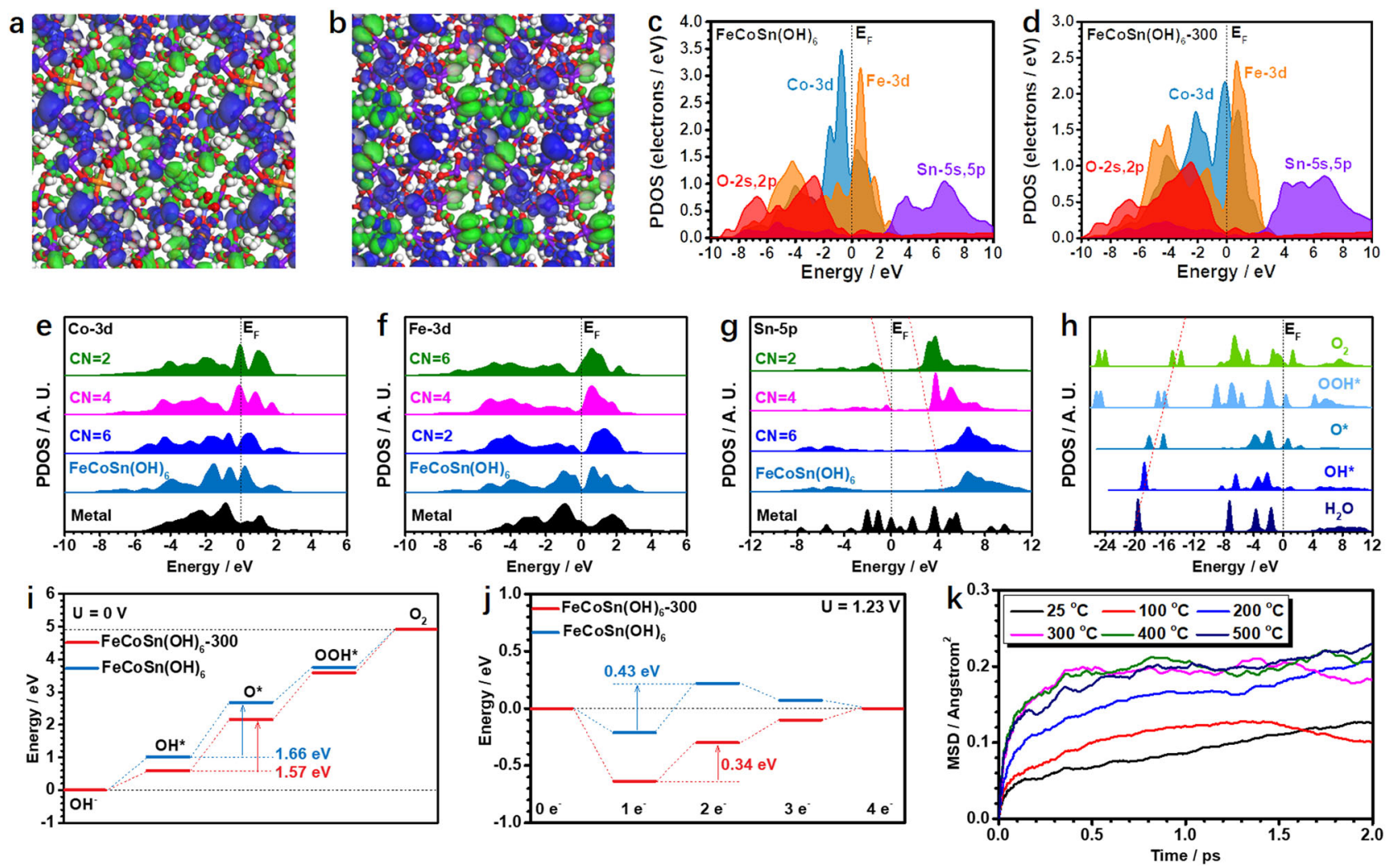

Fig. 5 DFT calculations of reaction process and amorphous process. The $3 \mathrm{D}$ contour plot of electronic distribution near Fermi level of a FeCoSn $(\mathrm{OH})_{6}$ and b FeCoSn $(\mathrm{OH})_{6}-300$. Blue balls: $\mathrm{Co}$, orange balls: Fe, purple balls: $\mathrm{Sn}$, red ball: $\mathrm{O}$, white balls: $\mathrm{H}$. Blue isosurface: bonding orbitals, and green isosurface: antibonding orbitals. The PDOS of $\mathbf{c} \mathrm{FeCoSn}(\mathrm{OH})_{6}$ and $\mathbf{d} \mathrm{FeCoSn}(\mathrm{OH})_{6}-300$. e The site-dependent PDOS of e Co sites, $\mathbf{f}$ Fe sites, and $\mathbf{g} \mathrm{Sn}-5 \mathrm{p}$ sites. h PDOS of key adsorbates in OER on amorphous FeCoSn $(\mathrm{OH})_{6}-300$. $\mathbf{i}$ The energetic trend of OER at $U=0 \mathrm{~V}$. $\mathbf{j}$ The energetic trend of OER at $U=1.23 \mathrm{~V}$. k The mean square displacements (MSD) of $\mathrm{FeCoSn}(\mathrm{OH})_{6}$ at different temperatures.

Fig. 26), which agrees with the previous reports ${ }^{29,51}$. The above results suggest that the amorphization $\mathrm{FeCoSn}(\mathrm{OH})_{6}$ can significantly enhance the OER performance (Supplementary Table 3).

Theoretical calculation of reaction process and amorphous process. To understand the superior OER performance of the amorphous $\mathrm{FeCoSn}(\mathrm{OH})_{6}$, density functional theory (DFT) calculations were performed to study the electronic modulations and the reaction trends (Supplementary Fig. 27, see more discussion in Methods section). With respect to $\mathrm{FeCoSn}(\mathrm{OH})_{6}$, it is noted that the surface Co sites with unsaturated coordination mainly contribute to the bonding orbitals, while the $\mathrm{Co}$ sites and $\mathrm{OH}$ groups mainly contribute to the antibonding orbitals (Fig. 5a). The contributions of $\mathrm{Sn}$ sites are limited in both bonding and antibonding orbitals near the Fermi level $\left(\mathrm{E}_{\mathrm{F}}\right)$. After amorphization, the surface of $\mathrm{FeCoSn}(\mathrm{OH})_{6}-300$ is electron-rich due to the presences of low-coordinated Co and Fe sites (Fig. 5b). In this case, the antibonding orbitals are dominated by the $\mathrm{OH}$ groups. Moreover, the orbital coupling of the surface $\mathrm{OH}$ groups may facilitate the electron transfer. The projected partial density of states (PDOS) have further proved the electronic modulations (Fig. 5c). For $\mathrm{FeCoSn}(\mathrm{OH})_{6}, \mathrm{Co}-3 \mathrm{~d}$ shows a sharp peak at $\mathrm{E}_{\mathrm{V}}-0.73 \mathrm{eV}\left(\mathrm{E}_{\mathrm{V}}=0 \mathrm{eV}\right)$ with the $\mathrm{e}_{\mathrm{g}}-\mathrm{t}_{2 \mathrm{~g}}$ splitting of $1.12 \mathrm{eV}$. Fe-3d orbitals suggest the significant contribution of the electron density near $\mathrm{E}_{\mathrm{F}}$ with the $\mathrm{e}_{\mathrm{g}}-\mathrm{t}_{2 \mathrm{~g}}$ splitting of $1.69 \mathrm{eV}$. The Sn-5p orbitals locate above the $\mathrm{E}_{\mathrm{F}}$ while the $\mathrm{O}-2 \mathrm{~s}$ and $2 \mathrm{p}$ orbitals show the dominant peak at $\mathrm{E}_{\mathrm{V}}-2.83 \mathrm{eV}$, and therefore $\mathrm{O}$ can serve as the electron reservoir. For $\mathrm{FeCoSn}(\mathrm{OH})_{6}-300$, the Co-3d orbitals upshift towards the $\mathrm{E}_{\mathrm{F}}(-0.11 \mathrm{eV})$, and the $\mathrm{e}_{\mathrm{g}}-\mathrm{t}_{2 \mathrm{~g}}$ splitting of $\mathrm{Fe}$ 3d significantly increases to $1.98 \mathrm{eV}$ (Fig. 5d). Given that the dominant peak of Co-3d orbitals in the middle of $\mathrm{e}_{\mathrm{g}}-\mathrm{t}_{2 \mathrm{~g}}$ orbitals of Fe-3d and the upshift of $\mathrm{O}-2 \mathrm{~s}$ and $2 \mathrm{p}$ orbitals $\left(\mathrm{E}_{\mathrm{V}}-2.42 \mathrm{eV}\right)$, as well as the similar $\mathrm{Sn}-5 \mathrm{p}$ orbitals after the amorphization compared with $\mathrm{FeCoSn}(\mathrm{OH})_{6}$, the p-d couplings between Co and $\mathrm{Fe}$ sites may be strongly promoted. Moreover, we investigated the site-dependent electronic structures for the $\mathrm{FeCoSn}(\mathrm{OH})_{6}-300$ (Fig. 5e). For Co-3d sites, the decrease of coordination numbers $(\mathrm{CN})$ will lead to the increased electron density near the $\mathrm{E}_{\mathrm{F}}$ and thus promotes the electroactivity towards OER. In contrast, $\mathrm{Fe}-3 \mathrm{~d}$ orbitals deliver an opposite dependence of the electronic structures with respect to $\mathrm{CN}$ (Fig. 5f). The $\mathrm{e}_{\mathrm{g}}-\mathrm{t}_{2 \mathrm{~g}}$ splitting becomes larger with the increased $\mathrm{CN}$, indicating that the pinning effect may play a critical role to stabilize the valence states of Co active sites. For Sn sites, it is revealed that the decrease of $\mathrm{CN}$ leads to the downshift of $5 \mathrm{p}$ orbitals towards $\mathrm{E}_{\mathrm{F}}$ and the decrease of energy barriers for electron transfer (Fig. $5 \mathrm{~g}$ ). For the key intermediates, the $\mathrm{O}$-species from $\mathrm{H}_{2} \mathrm{O}^{*}$ to $\mathrm{O}_{2}$ show the linear correlation of the $\sigma$ components in 2 p orbitals, which guarantees the efficient conversion of intermediates to $\mathrm{O}_{2}$ during OER (Fig. 5h). Furthermore, the reaction trend of OER has been compared for the amorphous $\mathrm{FeCoSn}(\mathrm{OH})_{6}-300$ and crystallized FeCoS$\mathrm{n}(\mathrm{OH})_{6}$. Owing to the strong binding strength of $\mathrm{OH}^{*}$ on $\mathrm{FeCoSn}(\mathrm{OH})_{6}-300$ and $\mathrm{FeCoSn}(\mathrm{OH})_{6}$, the energy barriers for the conversion from $\mathrm{OH}^{*}$ to $\mathrm{O}^{*}$ (rate-determining step) are 1.57 and $1.66 \mathrm{eV}$, respectively (Fig. 5i). Further calculations imply that $\mathrm{OH}^{*}$ can spontaneously adsorb on the surfaces of $\mathrm{FeCoSn}(\mathrm{OH})_{6}$ 300 and $\mathrm{FeCoSn}(\mathrm{OH})_{6}$ when the equilibrium potential $(\mathrm{U}=1.23 \mathrm{~V})$ was applied (Fig. 5j). The energy barrier for the 
conversion of $\mathrm{OH}^{*}$ to $\mathrm{O}^{*}$ is $0.34 \mathrm{eV}$ for $\mathrm{FeCoSn}(\mathrm{OH})_{6}-300$, which is smaller than that for $\mathrm{FeCoSn}(\mathrm{OH})_{6}(0.43 \mathrm{eV})$, further confirming the enhanced electroactivity for OER over $\mathrm{FeCoSn}(\mathrm{OH})_{6^{-}}$ 300. Additionally, we have carried out the molecule dynamic (MD) simulations under temperatures from 298 to $773 \mathrm{~K}$ to understand the amorphization of $\mathrm{CoSn}(\mathrm{OH})_{6}$. As shown in Fig. 5k, the mean square displacement (MSD) results show that the overall atomic movements are limited at the temperature range of $298-373 \mathrm{~K}$. When the temperature was increased to $473 \mathrm{~K}$, the significant increase of MSD value suggests the initiation of amorphization. No obvious increase of the MSD value is observed when the temperature is increased from 573 to $773 \mathrm{~K}$, suggesting the completion of amorphization. MD results suggest the amorphization occurs at the temperature range of 473-573 K, being consistent with experimental observation.

\section{Discussion}

In summary, we have demonstrated a versatile top-down strategy for the fabrications of amorphous oxides via a low-temperature thermal treatment of perovskite hydroxide. The generality of this strategy has been validated by the amorphizations of various unitary hydroxides, binary, and ternary perovskite hydroxides. Detailed characterizations indicate that the amorphization process is realized by the variations of coordination environment during thermal treatment. Specifically, the octahedral $\mathrm{M}-\mathrm{OH}$ coordination in perovskite hydroxide evolves to tetrahedral $\mathrm{M}-\mathrm{O}$ coordination in amorphous oxides with the disappearance of the 2nd M-M during the amorphization process. Benefiting from the disordered structure and the regulated electronic properties of amorphous oxides, the ternary amorphous oxide of FeCoS$\mathrm{n}(\mathrm{OH})_{6}-300$ can sever as promising catalyst for alkaline OER with superior activity and stability to many reported catalysts. The obtained amorphous oxides can also exhibit excellent stability without crystallization even when the temperature is as high as $500^{\circ} \mathrm{C}$. DFT calculations imply that the amorphization can strongly promote the $\mathrm{p}-\mathrm{d}$ couplings between $\mathrm{Co}$ and $\mathrm{Fe}$ sites, which significantly decrease the energy barrier for the conversion from $\mathrm{OH}^{*}$ to $\mathrm{O}^{*}$, the rate-determining step for OER. Moreover, MD calculations confirm that the amorphization occurs at the temperature range of $473-573 \mathrm{~K}$, being consistent with experimental observation. This work not only provides a robust strategy for fabricating various amorphous oxides but also may shed new light on nanomaterials, catalysis, chemistry, and beyond.

\section{Methods \\ Chemicals. Magnesium chloride hexahydrate $\left(\mathrm{MgCl}_{2} \cdot 6 \mathrm{H}_{2} \mathrm{O}, 98 \%\right)$, calcium chloride $\left(\mathrm{CaCl}_{2}, 96 \%\right)$, manganese chloride tetrahydrate $\left(\mathrm{MnCl}_{2} \cdot 4 \mathrm{H}_{2} \mathrm{O}, 99 \%\right)$, zinc chloride $\left(\mathrm{ZnCl}_{2}, 98 \%\right)$, cobalt chloride hexahydrate $\left(\mathrm{CoCl}_{2} \cdot 6 \mathrm{H}_{2} \mathrm{O}, 99 \%\right)$, nickel chloride hexahydrate $\left(\mathrm{NiCl}_{2} \cdot 6 \mathrm{H}_{2} \mathrm{O}, 98 \%\right)$, cadmium chloride $\left(\mathrm{CdCl}_{2}, 99 \%\right)$, copper chloride dihydrate $\left(\mathrm{CuCl}_{2} \cdot 2 \mathrm{H}_{2} \mathrm{O}, 98 \%\right)$, stannic chloride pentahydrate $\left(\mathrm{SnCl}_{4} \cdot 5 \mathrm{H}_{2} \mathrm{O}, 98 \%\right)$, 2-aminoethanol $\left(\mathrm{HO}\left(\mathrm{CH}_{2}\right)_{2} \mathrm{NH}_{2}, 99.9 \%\right)$, potassium hydro- xide $(\mathrm{KOH}, 85 \%)$, and ethanol $\left(\mathrm{C}_{2} \mathrm{H}_{5} \mathrm{OH}, 99.7 \%\right)$ were obtained from Sinopharm Chemical Reagent Co. Ltd. Hydrogen hexachloroiridate (IV) hexahydrate $\left(\mathrm{H}_{2} \mathrm{IrCl}_{6} \cdot \mathrm{H}_{2} \mathrm{O}, 99 \%\right)$ was obtained from Alfa Aesar. Ferrous chloride tetrahydrate $\left(\mathrm{FeCl}_{2} \cdot 4 \mathrm{H}_{2} \mathrm{O}\right)$ was obtained from Strem. Nafion solution $(\sim 5 \mathrm{wt} . \%$ in a mixture of lower aliphatic alcohols and water) was obtained from Sigma-Aldrich. The carbon powder (XC72R) was purchased from Vulcan. The deionized water $\left(18 \mathrm{M} \Omega \mathrm{cm}^{-1}\right)$ used in all experiments was prepared by passing water through an ultrapure purification system.}

Syntheses of $\mathbf{M}(\mathbf{O H})_{2}$ and $\mathbf{M}(\mathbf{O H})_{2}-\mathbf{4 0 0} .0 .2 \mathrm{mmol} \mathrm{MCl}_{2}(\mathrm{M}=\mathrm{Co}$ and $\mathrm{Cd})$ was dissolved in $15 \mathrm{~mL} \mathrm{KOH}(0.1 \mathrm{M})$ solution under ultrasonication and then kept undisturbed for $12 \mathrm{~h}$ at room temperature. The resulting precipitation was washed with deionized water by three times and dried at $80^{\circ} \mathrm{C}$ in an oven for $12 \mathrm{~h}$. The $\mathrm{M}(\mathrm{OH})_{2}-400$ was prepared by calcining $\mathrm{M}(\mathrm{OH})_{2}$ at $400^{\circ} \mathrm{C}$ in $\mathrm{H}_{2} / \mathrm{Ar}(5 \mathrm{vol} . \%)$ for $1 \mathrm{~h}$.

Synthesis of binary MSn(OH) $)_{6}$ and ternary $\mathbf{M C o S n}(\mathbf{O H})_{6} .51 .6 \mathrm{mg} \mathrm{SnCl}_{4} \cdot 5 \mathrm{H}_{2} \mathrm{O}$ and $0.2 \mathrm{mmol} \mathrm{MCl}_{2}(\mathrm{M}=\mathrm{Mg}, \mathrm{Ca}, \mathrm{Mn}, \mathrm{Fe}, \mathrm{Co}, \mathrm{Ni}, \mathrm{Cu}, \mathrm{Cd}$, and $\mathrm{Zn})$ was dissolved in $15 \mathrm{~mL}$ deionized water, followed by dropwise adding $0.5 \mathrm{~mL} 2$-aminoethanol under ultrasonic treatment for $15 \mathrm{~min}$. Then, the mixture was kept undisturbed at room temperature for $12 \mathrm{~h}$. The resulting precipitation was washed with deionized water by three times and dried at $80^{\circ} \mathrm{C}$ in an oven for $12 \mathrm{~h}$. The protocol for synthesizing ternary $\mathrm{MCoSn}(\mathrm{OH})_{6}$ perovskite hydroxides is similar to that for binary $\mathrm{MSn}(\mathrm{OH})_{6}$ except for changing the amounts of corresponding precursors. The detailed parameters are listed in Supplementary Table 4.

Synthesis of amorphous $\mathbf{M S n}(\mathrm{OH})_{6}-\mathbf{3 0 0}$ and $\mathbf{M C o S n}(\mathrm{OH})_{6}-300$. The $\mathrm{MSn}(\mathrm{OH})_{6}-300$ or $\mathrm{MCoSn}(\mathrm{OH})_{6}-300$ were prepared by annealing $\mathrm{MSn}(\mathrm{OH})_{6}$ or $\mathrm{MCoSn}(\mathrm{OH})_{6}(\mathrm{M}=\mathrm{Mg}, \mathrm{Ca}, \mathrm{Mn}, \mathrm{Fe}, \mathrm{Co}, \mathrm{Ni}, \mathrm{Cu}, \mathrm{Cd}$, and $\mathrm{Zn})$ at $300^{\circ} \mathrm{C}$ in $\mathrm{H}_{2} / \mathrm{Ar}(5$ vol.\%) for $1 \mathrm{~h}$.

Synthesis of $\mathbf{F e}-\mathrm{CoO} / \mathrm{SnO}_{2}$ and $\mathbf{F e}-\mathrm{CoSn} . \mathrm{Fe}-\mathrm{CoO} / \mathrm{SnO}_{2}$ and $\mathrm{Fe}-\mathrm{CoSn}$ were prepared by annealing $\mathrm{FeCoSn}(\mathrm{OH})_{6}$ at $700^{\circ} \mathrm{C}$ for $1 \mathrm{~h}$ in Air and $\mathrm{H}_{2} / \mathrm{Ar}(5 \mathrm{vol} . \%)$, respectively.

Synthesis of $\mathrm{CdIr}(\mathrm{OH})_{6}$ and $\mathrm{CdIr}(\mathrm{OH})_{6}-\mathbf{3 0 0} .20 .4 \mathrm{mg} \mathrm{H}_{2} \mathrm{IrCl}_{6} \cdot \mathrm{H}_{2} \mathrm{O}$ was dissolved in $10 \mathrm{~mL} \mathrm{KOH}$ solution $(0.1 \mathrm{M})$, followed by adding $9.15 \mathrm{mg} \mathrm{CdCl} 2$ under ultrasonication and then kept undisturbed for $12 \mathrm{~h}$ at room temperature. The resulting precipitation was washed with deionized water by three times and dried at $80{ }^{\circ} \mathrm{C}$ in an oven for $12 \mathrm{~h}$ to obtain $\mathrm{CdIr}(\mathrm{OH})_{6}$. For the synthesis of $\mathrm{CdIr}(\mathrm{OH})_{6^{-}}$ $300, \mathrm{CdIr}(\mathrm{OH})_{6}$ was calcinated at $300^{\circ} \mathrm{C}$ in $\mathrm{H}_{2} / \mathrm{Ar}(5$ vol.\%) for $1 \mathrm{~h}$.

Characterization. Low-magnification TEM was acquired on a HITACHI HT7700 transmission electron microscope at an accelerating voltage of $120 \mathrm{kV}$. Highresolution TEM (HRTEM) and high-angle annular dark-field scanning TEM (HAADF-STEM) were conducted on a FEI Tecnai F20 transmission electron microscope at an acceleration voltage of $200 \mathrm{kV}$. X-ray diffraction (XRD) patterns were collected on a Shimadzu XRD-6000 X-ray diffractometer. Scanning electron microscopy energy-dispersive X-ray spectroscopy (SEM-EDS) spectra were obtained with a HITACHI S-4700 cold field emission scanning electron microscope. Fourier transform infrared spectra were recorded on a VERTEX 70 spectrometer (Bruker). Raman spectra were recorded on a Horiba HR800 Raman spectrometer using the $633 \mathrm{~nm}$ laser as the excitation source. X-ray photoelectron spectra were collected with an SSI S-Probe XPS Spectrometer. The carbon peak at $284.6 \mathrm{eV}$ was used as a reference for calibration. XAS data were collected at the TLS17C1 and TLS11A beamline of the National Synchrotron Radiation Research Center (NSRRC, Hsinchu, Taiwan), respectively. Data were processed according to standard procedures using the Demeter program package (Version 0.9.24) ${ }^{52}$.

Electrochemical measurements. The electrochemical measurements were performed by using a CHI 660E workstation (Chenhua, Shanghai) with a threeelectrode configuration. All the experiments were carried out at room temperature. Saturated calomel electrode and Graphite rod were used as the reference and counter electrode, respectively. The inks of different electrocatalysts were prepared by sonicating $2 \mathrm{mg}$ catalyst and $2 \mathrm{mg}$ carbon powder with $0.4 \mathrm{~mL}$ isopropanol and $15 \mu \mathrm{L}$ Nafion for $30 \mathrm{~min}$. The working electrode was then fabricated by dropping $40 \mu \mathrm{L}$ ink onto a glass carbon electrode (GCE) with a geometric area of $0.196 \mathrm{~cm}^{2}$ Linear-sweep voltammograms and chronopotentiometry measurements were carried out to study the catalytic activity and stability, respectively. The Linear-sweep voltammograms measurements were operated on a GCE, and the chronopotentiometry measurements were operated on a carbon paper $\left(0.25 \mathrm{~cm}^{2}\right)$. All the polarization curves were $95 \%$ iR corrected and the linear scan voltammetry at $5 \mathrm{mV} \mathrm{s}^{-1}$ for each sample.

ECSA calculations. The ECSA was calculated by the method of $\mathrm{C}_{\mathrm{dl}}$.

$$
\mathrm{ECSA}=\frac{C_{\mathrm{dl}}}{C_{\mathrm{s}}}
$$

where Cs is the specific capacitance of the sample or the capacitance of an atomically smooth planar surface of the material per unit area under identical electrolyte conditions. For our estimates of surface area, we use general specific capacitances of $\mathrm{Cs}=0.040 \mathrm{mF} \mathrm{cm}^{-2}$ in $1 \mathrm{M} \mathrm{KOH}$ based on typical reported values.

TOF calculations. For OER, the TOF value is usually calculated by the equation:

$$
\mathrm{TOF}=\frac{J \times A}{4 \times F \times n}
$$

where J is the current density after $95 \%$ iR corrected, A is the geometric area of the electrode $\left(0.196 \mathrm{~cm}^{2}\right)$, F is Faraday's constant and $\mathrm{n}$ is the molar number of active sites. In our study, we suppose Co and Fe as active sites for catalysts.

The surface area of each cobalt oxide:

$$
\mathrm{S}_{\text {atom }}=4.838 \times\left[\frac{11.63}{6.023 \times 10^{23}}\right]^{\frac{2}{3}} \mathrm{~cm}^{2} \text { atom }^{-1}
$$


The molar number of atoms exposed on the surface:

$$
\mathrm{n}=\frac{\mathrm{ECSA}}{3.478 \times 10^{-15} \mathrm{~cm}^{2} \text { atom }^{-1} \times \mathrm{N}_{\mathrm{A}}}
$$

The number $\mathrm{n}$ was estimated via the total loading mass, according to the equation:

$$
\mathrm{n}=\frac{\mathrm{m} \times \mathrm{N}_{\mathrm{A}}}{M_{w}}
$$

where $\mathrm{m}$ is the loading mass, $\mathrm{N}_{\mathrm{A}}$ is Avogadro's constant, and $\mathrm{M}_{\mathrm{w}}$ is the molecular weight of the catalysts.

Calculation Setup. DFT calculations were used to investigate the enhanced catalytic performance through the CASTEP packages ${ }^{53}$. For all the calculations, we selected the generalized gradient approximation (GGA) with Perdew-BurkeErnzerhof (PBE) to supply an accurate description of the exchange-correlation energy ${ }^{54-56}$. The plane-wave basis cutoff energy was set to $380 \mathrm{eV}$ based on the ultrasoft pseudopotentials. For all the geometry optimizations in this work, the Broyden-Fletcher-Goldfarb-Shannon (BFGS) algorithm is selected ${ }^{57}$. The k-points with coarse quality have been applied for all the energy minimizations. For all the geometry optimizations, the calculations have to satisfy the following convergence criteria that the Hellmann-Feynman forces on the atom should not exceed $0.001 \mathrm{eV} / \AA$, and the total energy difference and the inter-ionic displacement should be less than $5 \times 10^{-5} \mathrm{eV} /$ atom and $0.005 \AA$ /atom, respectively. We first established the $3 \times 3 \times 1$ supercell of cubic of FeCoSn $(\mathrm{OH})_{6}$ with $\mathrm{Co}$ and Fe occupying the same Co sites, which shows a ratio of 3:1. This structure composition is consistent with experiment characterizations. To construct the amorphous structure, the MD simulations have been performed on $\mathrm{FeCoSn}(\mathrm{OH})_{6}$ under the NVT conditions at $573 \mathrm{~K}$ to be consistent with the experimental condition. The time step was set as $1 \mathrm{fs}$ and the total simulation time is 5 ps with 5000 simulation steps to obtain the amorphous structure. As the simulation time increases, the structure starts to become amorphous. After the simulation finishes, we further carried out the geometry optimizations to obtain the stabilized amorphous structure as the $\mathrm{FeCoSn}(\mathrm{OH})_{6}-300$. We have cleaved four layers of the amorphous structure from the (001) facet. Meanwhile, to understand the amorphous transformation temperature, the MD simulations have been carried out under different temperatures from 298 to $673 \mathrm{~K}$ under the NVT conditions. The time step is $1 \mathrm{fs}$ and the total simulation time is 5 ps with 5000 simulation steps.

\section{Data availability}

The data generated in this study are provided in Source Data file. Source data are provided with this paper.

Received: 4 October 2021; Accepted: 20 January 2022;

Published online: 04 March 2022

\section{References}

1. Chen, Y. et al. Phase engineering of nanomaterials. Nat. Rev. Chem. 4, 243-256 (2020).

2. Simonov, A. \& Goodwin, A. L. Designing disorder into crystalline materials. Nat. Rev. Chem. 4, 657-673 (2020).

3. Smith, R. D. L. et al. Photochemical route for accessing amorphous metal oxide materials for water oxidation catalysis. Science 340, 60-63 (2013).

4. Liu, Y. H. et al. Super plastic bulk metallic glasses at room temperature. Science 315, 1385-1388 (2007)

5. Luo, Q., Zhao, D. Q., Pan, M. X. \& Wang, W. H. Magnetocaloric effect in Gdbased bulk metallic glasses. Appl. Phys. Lett. 89, 081914 (2006).

6. Hong, S. et al. Ultralow-dielectric-constant amorphous boron nitride. Nature 582, 511-514 (2020).

7. Yang, Y. et al. Determining the three-dimensional atomic structure of an amorphous solid. Nature 592, 60-64 (2021).

8. Yang, N. et al. Novel amorphous/crystalline hetero-phase Pd nanosheets: one pot synthesis and highly selective hydrogenation reaction. Adv. Mater. 30, 1803234 (2018).

9. Wang, Y. et al. Tension-compression asymmetry in amorphous silicon. Nat. Mater. 20, 1371-1377 (2021).

10. Wang, B., Biesold, G. M., Zhang, M. \& Lin, Z. Q. Amorphous inorganic semiconductors for the development of solar cell, photoelectrocatalytic and photocatalytic applications. Chem. Soc. Rev. 50, 6914-6949 (2021).

11. Chen, G. et al. Two orders of magnitude enhancement in oxygen evolution reactivity on amorphous $\mathrm{Ba}_{0.5} \mathrm{Sr}_{0.5} \mathrm{Co}_{0.8} \mathrm{Fe}_{0.2} \mathrm{O}_{3-\delta}$ nanofilms with tunable oxidation state. Sci. Adv. 3, e1603206 (2017).

12. Wang, J. et al. Amorphization activated ruthenium-tellurium nanorods for efficient water splitting. Nat. Commun. 10, 5692 (2019).
13. Chen, G. et al. An amorphous nickel-iron-based electrocatalyst with unusual local structures for ultrafast oxygen evolution reaction. Adv. Mater. 31, 1900883 (2019).

14. Zhang, $X$. et al. Lithiation-induced amorphization of $\mathrm{Pd}_{3} \mathrm{P}_{2} \mathrm{~S}_{8}$ for highly efficient hydrogen evolution. Nat. Catal. 1, 460-468 (2018).

15. Lu, K. Nanocrystalline metals crystallized from amorphous solids: nanocrystallization, structure, and properties. Mater. Sci. Eng. R. Rep. 16, 161-221 (1996).

16. Zhao, H., Chen, X., Wang, G., Qiu, Y. \& Guo, L. Two-dimensional amorphous nanomaterials: synthesis and applications. 2D Mater. 6, 032002 (2019).

17. Buchholz, D. B. et al. The structure and properties of amorphous indium oxide. Chem. Mater. 26, 5401-5411 (2014).

18. Liu, J. Z. et al. Ultrathin amorphous cobalt-vanadium hydr(oxy)oxide catalysts for the oxygen evolution reaction. Energy Environ. Sci. 11, 1736-1741 (2018).

19. Duan, Y. X. et al. Amorphizing of $\mathrm{Cu}$ nanoparticles toward highly efficient and robust electrocatalyst for $\mathrm{CO}_{2}$ reduction to liquid fuels with high faradaic efficiencies. Adv. Mater. 30, 1706194 (2018).

20. Yao, Y. G. et al. Carbothermal shock synthesis of high-entropy-alloy nanoparticles. Science 359, 1489-1494 (2018).

21. Sun, Z. M. et al. Pressure-induced reversible amorphization and an amorphous-amorphous transition in $\mathrm{Ge}_{2} \mathrm{Sb}_{2} \mathrm{Te}_{5}$ phase-change memory material. Proc. Natl Acad. Sci. USA 108, 10410-10414 (2011).

22. Chance, W. M., Bugaris, D. E., Sefat, A. S. \& Loye, H. C. Z. Crystal growth of new hexahydroxometallates using a hydroflux. Inorg. Chem. 52, 11723-11733 (2013).

23. Albrecht, R., Hunger, J., Doert, T. \& Ruck, M. CaNa[Cr(OH) 6 -A layered hydroxochromate (III) with ordered brucite structure and its thermal decomposition. Z. Anorg. Allg. Chem. 646, 1-9 (2020).

24. Jena, H., Kutty, K. V. G. \& Kutty, T. R. N. Ionic transport and structural investigations on $\mathrm{MSn}(\mathrm{OH})_{6}(\mathrm{M}=\mathrm{Ba}, \mathrm{Ca}, \mathrm{Mg}, \mathrm{Co}, \mathrm{Zn}, \mathrm{Fe}, \mathrm{Mn})$ hydroxide perovskites synthesized by wet sonochemical methods. Mater. Chem. Phys. $\mathbf{8 8}$ 167-179 (2004).

25. Zeng, J. et al. Transformation process and photocatalytic activities of hydrothermally synthesized $\mathrm{Zn} 2 \mathrm{SnO} 4$ nanocrystals. J. Phys. Chem. C. 112, 4159-4167 (2008)

26. May, K. J. et al. Influence of oxygen evolution during water oxidation on the surface of perovskite oxide catalysts. J. Phys. Chem. Lett. 3, 3264-3270 (2012).

27. Yang, J., Liu, H. W., Martens, W. N. \& Frost, R. L. Synthesis and characterization of cobalt hydroxide, cobalt oxyhydroxide, and cobalt oxide nanodiscs. J. Phys. Chem. C. 114, 111-119 (2010).

28. Ju, D. X. et al. Near room temperature, fast-response, and highly sensitive triethylamine sensor assembled with $\mathrm{Au}$-loaded $\mathrm{ZnO} / \mathrm{SnO}_{2}$ core-shell nanorods on flat alumina substrates. ACS Appl. Mater. Interfaces 7, 19163-19171 (2015).

29. Mclntyre, N. S. \& Cook, M. G. X-ray photoelectron studies on some oxides and hydroxides of cobalt, nickel, and copper. Anal. Chem. 47, 2208-2213 (1975).

30. Zhuang, L. Z. et al. Ultrathin iron-cobalt oxide nanosheets with abundant oxygen vacancies for the oxygen evolution reaction. Adv. Mater. 29, 1606793 (2017).

31. Inagaki, M., Kuroishi, T., Yamashita, Y. \& Urata, M. Syntheses of MSn $(\mathrm{OH})_{6}$ by coprecipitation and of $\mathrm{MSnO}_{3}$ by thermal decomposition $(\mathrm{M}=\mathrm{Mg}, \mathrm{Co}$, $\mathrm{Zn}, \mathrm{Mn}, \mathrm{Cd}, \mathrm{Ca}, \mathrm{Sr}, \mathrm{Ba})$. Z. Anorg. Allg. Chem. 527, 193-202 (1986).

32. Yang, J. Y. et al. Charge transfer induced multifunctional transitions with sensitive pressure manipulation in a metal-organic framework. Inorg. Chem. 54, 6433-6438 (2015).

33. Agrestini, S. et al. Nature of the magnetism of iridium in the double perovskite Sr2CoIrO6. Phys. Rev. B 100, 014443 (2019).

34. Guan, D. et al. Exceptionally robust face-sharing motifs enable efficient and durable water oxidation. Adv. Mater. 33, 2103392 (2021).

35. Guan, D. Q. et al. Utilizing ion leaching effects for achieving high oxygenevolving performance on hybrid nanocomposite with self-optimized behaviors. Nat. Commun. 11, 3376 (2020).

36. Burnus, T. et al. X-ray absorption and X-ray magnetic dichroism study on $\mathrm{Ca}_{3} \mathrm{CoRhO}_{6}$ and $\mathrm{Ca}_{3} \mathrm{FeRhO}_{6}$. Phys. Rev. B 77, 205111 (2008).

37. Chin, Y. Y. et al. Spin-orbit coupling and crystal-field distortions for a lowspin 3d5 state in $\mathrm{BaCoO}_{3}$. Phys. Rev. B 100, 250139 (2019).

38. $\mathrm{Hu}, \mathrm{Z}$. W. et al. Spin-state order/disorder and metal-insulator transition in $\mathrm{GdBaCo}_{2} \mathrm{O}_{5.5}$ : experimental determination of the underlying electronic structure. N. J. Phys. 14, 123025 (2012).

39. Chen, J. M. et al. A complete high-to-low spin state transition of trivalent cobalt ion in octahedral symmetry in $\mathrm{SrCo}_{0.5} \mathrm{Ru}_{0.5} \mathrm{O}_{3-\delta}$. J. Am. Chem. Soc. 136, 1514-1519 (2014)

40. Burnus, T. et al. Valence, spin, and orbital state of Co ions in one-dimensional $\mathrm{Ca}_{3} \mathrm{Co}_{2} \mathrm{O}_{6}$ : An X-ray absorption and magnetic circular dichroism study. Phys. Rev. B 74, 245111 (2006). 
41. Chang, C. F. et al. Spin blockade, orbital occupation, and charge ordering in $\mathrm{La}_{1.5} \mathrm{Sr}_{0.5} \mathrm{CoO}_{4}$. Phys. Rev. Lett. 102, 116401 (2009).

42. Hollmann, $\mathrm{N}$. et al. Electronic and magnetic properties of the kagome systems $\mathrm{YBaCo}_{4} \mathrm{O}_{7}$ and $\mathrm{YBaCo}_{3} \mathrm{MO}_{7}(\mathrm{M}=\mathrm{Al}, \mathrm{Fe})$. Phys. Rev. B 80, 085111 (2009).

43. Lin, F. et al. Synchrotron X-ray analytical techniques for studying materials electrochemistry in rechargeable batteries. Chem. Rev. 117, 13123-13186 (2017).

44. Chen, Y. J. et al. Isolated single iron atoms anchored on $\mathrm{N}$-doped porous carbon as an efficient electrocatalyst for the oxygen reduction reaction. Angew. Chem. Int. Ed. 56, 6937-6941 (2017)

45. Cao, L. L. et al. Identification of single-atom active sites in carbon-based cobalt catalysts during electrocatalytic hydrogen evolution. Nat. Catal. 2, 134-141 (2019).

46. Jiao, J. Q. et al. Copper atom-pair catalyst anchored on alloy nanowires for selective and efficient electrochemical reduction of $\mathrm{CO}_{2}$. Nat. Chem. 11, 222-228 (2019).

47. Bergmann, A. et al. Reversible amorphization and the catalytically active state of crystalline $\mathrm{Co}_{3} \mathrm{O}_{4}$ during oxygen evolution. Nat. Commun. 6, 8625 (2015).

48. McCrory, C. C. L., Jung, S., Peters, J. C. \& Jaramillo, T. F. Benchmarking heterogeneous electrocatalysts for the oxygen evolution reaction. J. Am. Chem. Soc. 135, 16977-16987 (2013).

49. Ma, R. G. et al. Ultrafine molybdenum carbide nanoparticles composited with carbon as a highly active hydrogen-evolution electrocatalyst. Angew. Chem. Int. Ed. 54, 14723-14727 (2015).

50. Li, B. Q. et al. Regulating p-block metals in perovskite nanodots for efficient electrocatalytic water oxidation. Nat. Commun. 8, 934 (2017).

51. Song, F., Schenk, K. \& Hu, X. L. A nanoporous oxygen evolution catalyst synthesized by selective electrochemical etching of perovskite hydroxide $\mathrm{CoSn}(\mathrm{OH})_{6}$ nanocubes. Energy Environ. Sci. 9, 473-477 (2016)

52. Ravel, B. \& Newville, M. ATHENA, ARTEMIS, HEPHAESTUS: Data analysis for X-ray absorption spectroscopy using IFEFFIT. J. Synchrotron Radiat. 12, 537-541 (2005).

53. Clark, S. J. et al. First principles methods using castep. Z. Fur Kristallographie 220, 567-570 (2005).

54. Perdew, J. P., Burke, K. \& Ernzerhof, M. Generalized gradient approximation made simple. Phys. Rev. Lett. 77, 3865-3868 (1996).

55. Hasnip, P. J. \& Pickard, C. J. Electronic energy minimisation with ultrasoft pseudopotentials. Comput. Phys. Commun. 174, 24-29 (2006).

56. Perdew, J. P. et al. Atoms, molecules, solids, and surfaces: applications of the generalized gradient approximation for exchange and correlation. Phy. Rev. B 46, 6671-6687 (1992).

57. Head, J. D. \& Zerner, M. C. A Broyden-Fletcher-Goldfarb-Shanno optimization procedure for molecular geometries. Chem. Phys. Lett. 122, 264-270 (1985).

\section{Acknowledgements}

This work was financially supported by the National Key R\&D Program of China (2020YFB1505802), the Ministry of Science and Technology of China (2017YFA0208200, 2016YFA0204100), the National Natural Science Foundation of China (22025108, 2212100020, 21905188, 51802206), Guangdong Provincial Natural Science Fund for Distinguished Young Scholars (2021B1515020081), Guangdong Provincial Key
Laboratory of Energy Materials for Electric Power (2018B030322001), the project of scientific and technologic infrastructure of Suzhou (SZS201708), the Priority Academic Program Development of Jiangsu Higher Education Institutions (PAPD), and the startup supports from Xiamen University and the Guangzhou Key Laboratory of Low Dimensional Materials and Energy Storage Devices (20195010002). We thank beamline TLS11A and TLS17C1 (National Synchrotron Radiation Research Center) for providing the beam time. We acknowledge support from the Max Planck-POSTECH-Hsinchu Center for Complex Phase Materials.

\section{Author contributions}

X.H. and Y.X. conceived and supervised the research. X.H., Y.X., S.L., S.G., Y.Z., and Q.S. designed the experiments. X.H., S.L., and G.R. performed most of the experiments and data analysis. B.H. conducted DFT calculations. X.H., Y.X., S.L., and L. L. wrote the paper. Z.H. and S.L. performed XAS analysis. J.L., Y.C., and Y.L. performed XAS testing. All authors discussed the results and commented on the paper.

\section{Competing interests}

The authors declare no competing interests.

\section{Additional information}

Supplementary information The online version contains supplementary material available at https://doi.org/10.1038/s41467-022-28888-3.

Correspondence and requests for materials should be addressed to Bolong Huang, Yong $\mathrm{Xu}$ or Xiaoqing Huang.

Peer review information Nature Communications thanks Min-Rui Gao, Jing-Li Luo and the other, anonymous, reviewer for their contribution to the peer review of this work.

Reprints and permission information is available at http://www.nature.com/reprints

Publisher's note Springer Nature remains neutral with regard to jurisdictional claims in published maps and institutional affiliations.

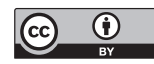

Open Access This article is licensed under a Creative Commons Attribution 4.0 International License, which permits use, sharing, adaptation, distribution and reproduction in any medium or format, as long as you give appropriate credit to the original author(s) and the source, provide a link to the Creative Commons license, and indicate if changes were made. The images or other third party material in this article are included in the article's Creative Commons license, unless indicated otherwise in a credit line to the material. If material is not included in the article's Creative Commons license and your intended use is not permitted by statutory regulation or exceeds the permitted use, you will need to obtain permission directly from the copyright holder. To view a copy of this license, visit http://creativecommons.org/ licenses/by/4.0/.

(C) The Author(s) 2022 